\title{
Alternative Lengthening of Telomeres (ALT) in Pancreatic Neuroendocrine Tumors: Ready for Prime-Time in Clinical Practice?
}

\author{
Claudio Luchini ${ }^{1} \cdot$ Rita T. Lawlor ${ }^{2}$ - Samantha Bersani ${ }^{2}$ - Caterina Vicentini ${ }^{2} \cdot$ Gaetano Paolino $^{2} \cdot$ Paola Mattiolo $^{1}$. \\ Antonio Pea ${ }^{3}$ - Sara Cingarlini ${ }^{4} \cdot$ Michele Milella $^{4}$ - Aldo Scarpa ${ }^{1,2}$ (D)
}

Accepted: 20 May 2021 / Published online: 16 July 2021

(C) The Author(s) 2021

\begin{abstract}
Purpose of Review Alternative lengthening of telomeres (ALT) is a telomerase-independent mechanism used by some types of malignancies, including pancreatic neuroendocrine tumors, to overcome the issue of telomere shortening, thus supporting tumor growth and cell proliferation. This review is focused on the most important achievements and opportunities deriving from ALT assessment in PanNET onco-pathology, highlighting the most promising fields in which such biomarker could be implemented in clinical practice.

Recent Findings In pancreatic neuroendocrine tumors (PanNET), ALT is strongly correlated with the mutational status of two chromatin remodeling genes, DAXX and ATRX. Recent advances in tumor biology permitted to uncover important roles of ALT in the landscape of PanNET, potentially relevant for introducing this biomarker into clinical practice. Indeed, ALT emerged as a reliable indicator of worse prognosis for PanNET, helping in clinical stratification and identification of "high-risk" patients. Furthermore, it is a very specific marker supporting the pancreatic origin of neuroendocrine neoplasms and can be used for improving the diagnostic workflow of patients presenting with neuroendocrine metastasis from unknown primary. The activation of this process can be determined by specific FISH analysis.

Summary ALT should be introduced in clinical practice for identifying "high-risk" PanNET patients and improving their clinical management, and as a marker of pancreatic origin among neuroendocrine tumors.
\end{abstract}

Keywords Pancreatic neuroendocrine tumors $\cdot$ PanNET $\cdot$ ALT $\cdot$ DAXX $\cdot$ ATRX

\section{Introduction}

Since somatic cells are unable to polymerize all DNA during physiological replication, each round of cell division

This article is part of the Topical Collection on Neuroendocrine Neoplasms

Aldo Scarpa

aldo.scarpa@univr.it

1 Department of Diagnostics and Public Health, Section of Pathology, University and Hospital Trust of Verona, 37134 Verona, Italy

2 ARC-Net Research Centre, University and Hospital Trust of Verona, 37134 Verona, Italy

3 Department of Surgery, University and Hospital Trust of Verona, Verona, Italy

4 Department of Medicine, Section of Oncology, University and Hospital Trust of Verona, Verona, Italy determines telomere shortening $[1,2]$. This mechanism is the same in cancer cells, where it is used to support cell proliferation and the consequent tumor growth [3-5]. Thus, a tumor can achieve a remarkable replicative capacity also thanks to the activation of this telomere maintenance mechanism [3-5].

Physiologically, telomere shortening is counterbalanced by the specific activity of the enzyme telomerase, which is a DNA polymerase that uses reverse transcription of a RNA template to produce de novo synthesis of telomeric DNA [5]. Interestingly, some neoplasms do not overcome the issue of telomere shortening by using telomerase, but maintain telomere length with a different process, which is totally telomerase-independent and called alternative lengthening of telomeres (ALT) [6,7]. Differently from the telomerasedependent mechanism, in ALT, the telomeric DNA is produced on the basis of a DNA template [6]. Indeed, the copy template can either be represented by the telomere itself, by a telomere on a sister chromatid, or even by another 
chromosome [8]. Due to these different mechanisms, ALT cells usually show long and heterogeneous telomeres with sub-nuclear formations, the ALT-associated promyelocytic leukemia bodies (APB), which comprise telomeric DNA and the telomere-specific binding proteins, also known as terminal restriction fragments (TRF) [8]. Thanks to these features, ALT status can be easily determined by specific FISH analysis (Fig. 1).

ALT has been detected in different tumor types, including soft-tissue sarcomas and brain tumors $[9,10]$. In soft-tissue sarcomas, a recent meta-analysis analyzing 551 patients with different types of sarcomas demonstrated that activation of the ALT mechanism was strongly associated with an increased risk of mortality (doubled risk of mortality, HR = 2.02) [11•]. The only exception was represented by osteosarcoma, in which ALT did not show a clear and reliable biologic significance. Notably, although ALT was associated with a higher mitotic count and tumor grade in this type of malignancies, it maintained its prognostic significance also using data from multivariable analysis, highlighting its prognostic relevance [11•].

In brain tumors, ALT appears as the major telomere maintenance mechanism in $I D H 1$-mutated astrocytomas [12]. Furthermore, the activation of the ALT mechanism emerged as a common process associated to the metastatic behavior of primary medulloblastomas [13]. These findings highlight both the biological importance of ALT in tumor progression and its broad prognostic significance.

Interestingly, ALT has also been detected in pancreatic neuroendocrine tumors (PanNET), with important diagnostic and prognostic implications. Here we will analyze and discuss more in depth the most important findings related to ALT in PanNET, shedding light on its potential applications in clinical practice.

\section{ALT in Pancreatic Neuroendocrine Tumors}

\section{ALT Is Strictly Associated with DAXXIATRX Mutations}

The first description of the presence of the ALT process in a significant proportion of PanNET was provided by Marinoni et al. in 2014 [14••]. The authors demonstrated that the activation of such process was strictly correlated with a malignant behavior, including a higher rate of distant metastasization. Notably, the authors also described a strong association of ALT with the mutational status of two chromatin remodeling genes, whose mutations are usually mutually exclusive: death domain-associated protein $(D A X X)$ and $\alpha$-thalassemia/mental retardation X-linked $(A T R X)$ genes [14॰•]. These genes encode homonymous proteins that exert their function in the nucleus, by regulating the deposition of histone variant $\mathrm{H} 3.3$ during the assembly of peri-centromeric and telomeric chromatin [15]. Subsequent studies on the molecular landscape of PanNET confirmed the presence of ALT mechanism as a frequently biological process activated in this tumor type, and also its association with $D A X X / A T R X$ mutations [16•, $17,18 \bullet \bullet, 19]$. In general, $D A X X$ mutations were more commonly reported than $A T R X$ mutations in PanNET [16•, 17, $18 \bullet \bullet, 19]$, whereas those affecting $A T R X$ were markedly prominent in sarcomas [11•].

Of importance, the presence of DAXX/ATRX mutations can be studied not only with a direct molecular analysis but also with immunohistochemistry, which represents a reliable surrogate of their mutational status. Indeed, in the case of mutation, there is a total loss of expression of the corresponding protein at the immunohistochemical level (Fig. 2) [16•].

\section{ALT Is a Strong Prognostic Indicator in PanNET}

In PanNET, the first description of the poor prognostic role of the activated ALT mechanism has been provided by Marinoni and colleagues that analyzed both cancer-specific and diseasefree survival $[14 \bullet \bullet]$. These observations were further confirmed by different studies analyzing a broad spectrum of prognostic indexes $[16 \bullet, 17,18 \bullet \bullet, 20,21 \bullet \bullet, 22 \bullet]$, highlighting the clinical value of this biomarker. In particular, the studies of Scarpa et al. that analyzed 102 PanNET and of Singhi et al. that analyzed 321 PanNET were the first reports that extensively analyzed and described the poor prognostic value of ALT also in G2 PanNET [18••]. Furthermore, Scarpa et al. showed that, in both PanNET G1 and G2, the activated ALT
Fig. 1 A classic example of a pancreatic neuroendocrine tumor with the activation of the ALT mechanism analyzed with telomere-specific FISH. The presence of large ultrabright telomere FISH signals (arrows) is the pictorial marker of the activated alternative lengthening of telomere mechanism $(\mathbf{A} \times 4$ original magnification; $\mathbf{B} \times 10$ )
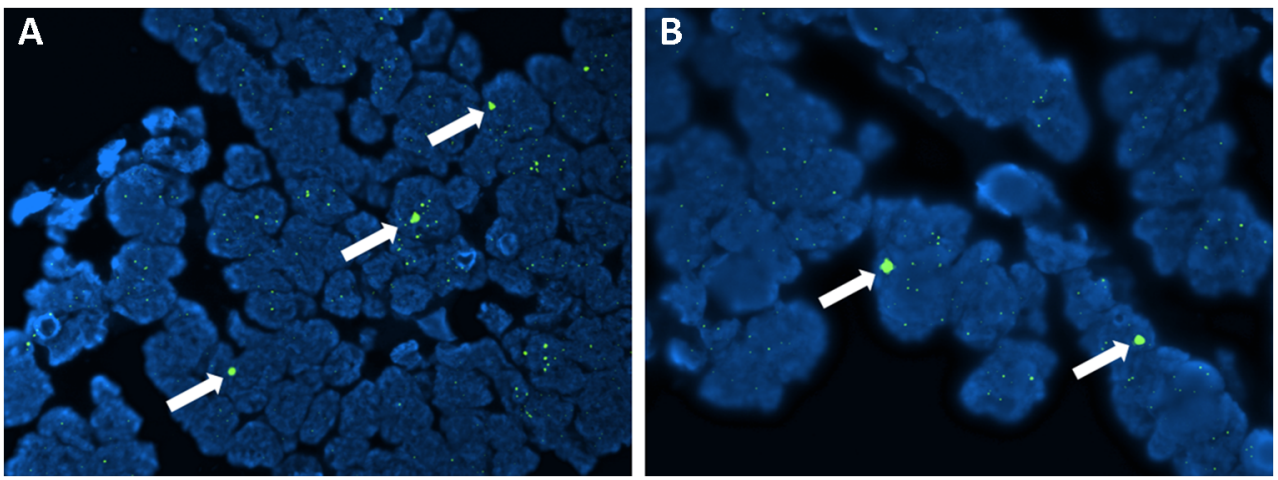


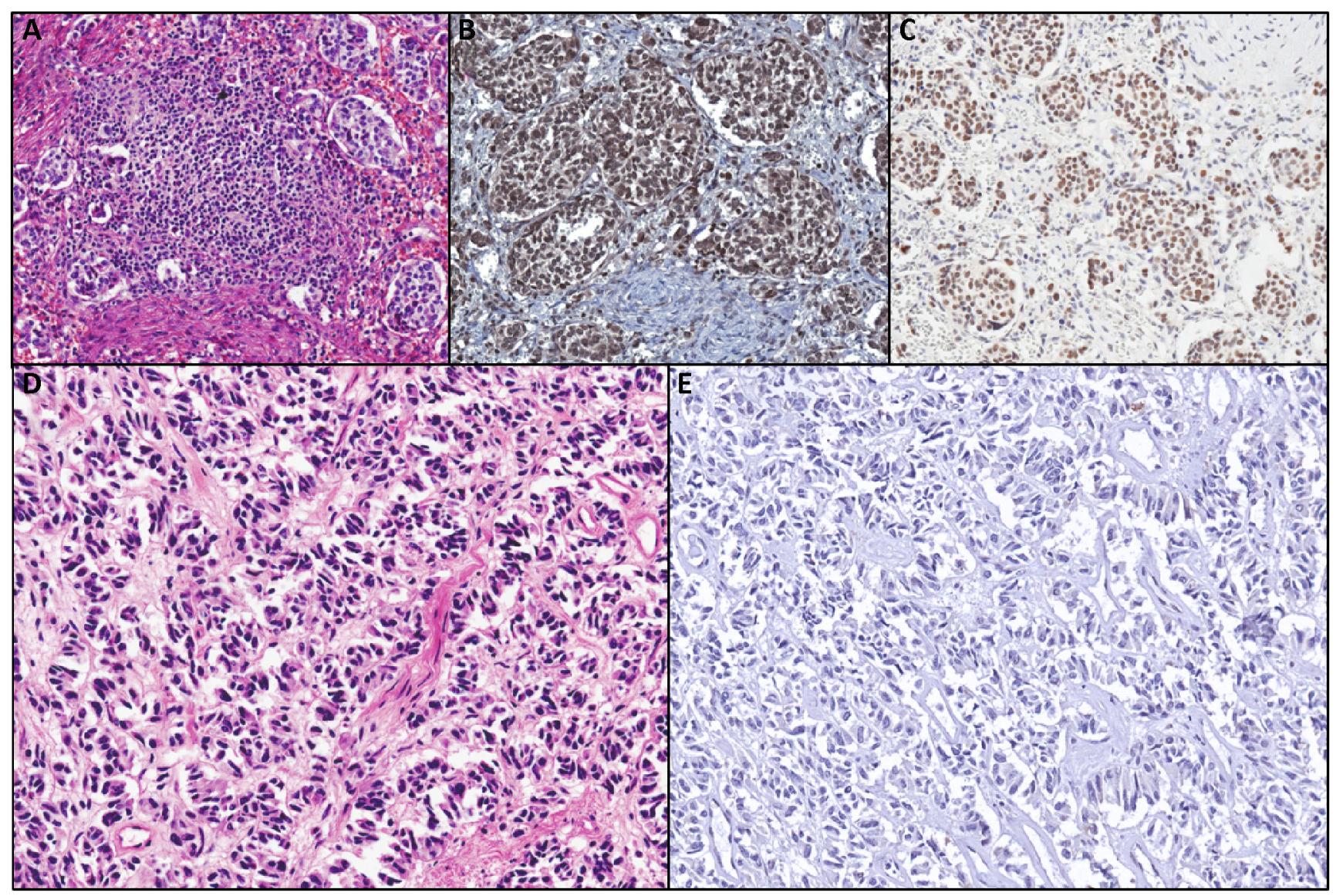

Fig. 2 Representation of two paradigmatic cases of pancreatic neuroendocrine tumors, one with retained expression of DAXX/ATRX at immunohistochemistry (A hematoxylin-eosin, B DAXX immunostaining, C ATRX immunostaining; original magnification $\times 10)$ and one with DAXX loss (D hematoxylin-eosin, E DAXX immunostaining, highlighting loss of nuclear expression; original magnification $\times 20$ ) mechanism was also associated with a recurrent pattern of whole chromosomal loss, which typically involved specific chromosomes, comprising chromosomes $1,2,3,6,8,10$, $11,15,16$, and $22[18 \cdot \bullet]$. All considered, ALT mechanism, $D A X X / A T R X$ mutations, and specific chromosome loss compose a so-called ALT phenotype that demonstrated a poor prognostic impact in PanNET patients.

Kim et al. also correlated the ALT phenotype with a worse prognosis, and in particular with a reduced recurrence-free survival; furthermore, they highlighted that such feature was specifically correlated with specific histological parameters [21••]. Indeed, studying a cohort of 269 patients, they reported a strong association of ALT with tumor size, nodal metastasis, and vascular and perineural invasion $[21 \bullet \cdot$. Notably, in their report, the authors analyzed ALT status also in G3 tumors, reporting the ALT activation in 2 of 8 high-grade pancreatic neoplasms. Interestingly, when limiting to patients with distant metastases, those with ALT-positive primary tumors had significantly better overall survival, further refining the knowledge on the prognostic role of ALT in PanNET.
Of interest is also a recent study of Pea and colleagues, which tested the prognostic power of ALT in a series of small PanNET, using $3 \mathrm{~cm}$ as an arbitrary threshold for considering a PanNET as a small lesion [16॰]. As prognostic index, the authors analyzed the risk of liver metastasization. The cohort was composed of 87 small PanNET, and the presence of the ALT phenotype was confirmed as a poor prognostic indicator also in small lesions.

A last insight regarding the potential clinical value of ALT in PanNET has been provided by Hackeng et al. that focused their analysis on a cohort of 31 patients with insulinomas and found that ALT phenotype was strictly associated with the risk of developing liver metastasis [22•].

The fact that all findings on the poor prognostic impact of ALT are overlapping in different PanNET cohorts, including large and small lesions, G1 and G2 tumors, and nonfunctioning neoplasms and insulinomas, highlights that this biomarker can be used as a reliable prognostic indicator in PanNET patients. Its determination during the routine diagnostic workflow, above all for patients with small and nonfunctioning lesions, can help in selected patients for upfront 
surgery (ALT positive) vs. active surveillance (ALT negative).

\section{Other Insights on ALT in PanNET}

A recent study assessed the feasibility of ALT determination on materials from fine needle aspiration (FNA), as well as of immunohistochemistry in determining DAXX and ATRX expression [23]. Investigating 65 PanNET, VandenBussche et al. established that both ALT status and DAXX/ATRX expression can be accurately performed on FNA specimens. The opportunity to determine ALT in small biospecimens has important implications for the introduction of these biomarkers in clinical practice. Thank to this possibility, indeed, ALT status and DAXX/ATRX expression may represent a decisive tool in the pre-surgical setting, helping in the identification of patients with "high-risk" lesions, thus improving PanNET clinical management.

In the case of neuroendocrine metastasis from unknown primary, which represents a non-negligible proportion of cases, Dogeas et al. indicated that the presence of the activated ALT mechanism could represent an important tool in supporting the pancreatic origin of a neuroendocrine tumor [24]. Indeed, analyzing a cohort of 90 gastroenteropancreatic NET, they found that the specificity of ALT for detecting pancreatic origin was very high (96\%). Also, this feature should be taken into account for implementing the possibility of ALT determination in clinical practice.

A last insight regards the possibility to predict the ALT status on the basis of radiological findings. McGovern and colleagues showed that some features identified at computed tomography were significantly associated with the ALT phenotype, such as tumor necrosis, vascular invasion, pancreatic duct dilation, and liver metastasization. These findings should be read in the context of multidisciplinary and precision medicine, where both pathology and radiology can integrate their information guiding the best clinical management of PanNET patients.

\section{Conclusions}

The assessment of the ALT status can be very useful to improve the clinical practice for PanNET patients: (i) to stratify prognosis of resected patients, with immediate implications for patients management; (ii) in the pre-surgical setting, serving as an additional tool to select patients for surgical resection; and (iii) as a marker of pancreatic origin, improving the diagnostic workflow of patients presenting with neuroendocrine metastasis from unknown primary. Globally considered, these represent three major reasons that call for launching ALT determination in the PanNET clinical practice.
Author Contribution All authors conceived the study, review the literature, wrote the manuscript, and approved the current version of the manuscript for the submission.

Funding Open access funding provided by Università degli Studi di Verona within the CRUI-CARE Agreement. This study is supported by Associazione Italiana Ricerca sul Cancro (AIRC 5x1000 n. 12182), Fondazione Cariverona: Oncology Biobank Project "Antonio Schiavi" (prot. 203885/2017), and Fondazione Italiana Malattie Pancreas (FIMP, Ministero Salute, J38D19000690001).

Availability of Data and Material All data are available in the manuscript.

Code Availability Not applicable.

\section{Declarations}

Ethics Approval Not applicable (review).

Consent to Participate Not applicable (review).

Consent for Publication Not applicable (review).

Conflict of Interest Claudia Luchini has received compensation from BioScience Communications for expert testimony on microsatellite instability. Rita T. Lawlor declares that she has no conflict of interest. Samantha Bersani declares that she has no conflict of interest. Caterina Vicentini declares that she has no conflict of interest. Gaetano Paolino declares that he has no conflict of interest. Paola Mattiolo declares that she has no conflict of interest. Antonio Pea declares that he has no conflict of interest. Sara Cingarlini declares that she has no conflict of interest. Michele Milella declares that he has no conflict of interest. Aldo Scarpa declares that he has no conflict of interest.

Open Access This article is licensed under a Creative Commons Attribution 4.0 International License, which permits use, sharing, adaptation, distribution and reproduction in any medium or format, as long as you give appropriate credit to the original author(s) and the source, provide a link to the Creative Commons licence, and indicate if changes were made. The images or other third party material in this article are included in the article's Creative Commons licence, unless indicated otherwise in a credit line to the material. If material is not included in the article's Creative Commons licence and your intended use is not permitted by statutory regulation or exceeds the permitted use, you will need to obtain permission directly from the copyright holder. To view a copy of this licence, visit http://creativecommons.org/licenses/by/4.0/.

\section{References}

Papers of particular interest, published recently, have been highlighted as:

- Of importance

•- Of major importance

1. Allsopp RC, Harley CB. Evidence for a critical telomere length in senescent human fibroblasts. Exp Cell Res. 1995;219:130-6.

2. Allsopp RC, Chang E, Kashefi-Aazam M, Rogaev EI, Piatyszek MA, Shay JW, et al. Telomere shortening is associated with cell division in vitro and in vivo. Exp Cell Res. 1995;220:194-200. 
3. Gaspar TB, Sá A, Lopes JM, Sobrinho-Simões M, Soares P, Vinagre J. Telomere maintenance mechanisms in cancer. Genes (Basel). 2018;9.

4. Shay JW, Zou Y, Hiyama E, Wright WE. Telomerase and cancer. Hum Mol Genet. 2001;10:677-85.

5. Granger MP, Wright WE, Shay JW. Telomerase in cancer and aging. Crit Rev Oncol Hematol. 2002;41:29-40.

6. Heaphy CM, Subhawong AP, Hong SM, Goggins MG, Montgomery EA, Gabrielson E, et al. Prevalence of the alternative lengthening of telomeres telomere maintenance mechanism in human cancer subtypes. Am J Pathol. 2011;179:1608-15.

7. Henson JD, Neumann AA, Yeager TR, Reddel RR. Alternative lengthening of telomeres in mammalian cells. Oncogene. 2002;21:598-610.

8. Yeager TR, Neumann AA, Englezou A, Huschtscha LI, Noble JR, Reddel RR. Telomerase-negative immortalized human cells contain a novel type of promyelocytic leukemia (PML) body. Cancer Res. 1999;59:4175-9.

9. Rosenberg AE. WHO Classification of Soft Tissue and Bone, fourth edition: summary and commentary. Curr Opin Oncol. 2013;25:571-3.

10. Sugarman ET, Zhang G, Shay JW. In perspective: An update on telomere targeting in cancer. Mol Carcinog. 2019;58:1581-8.

11. Lawlor RT, Veronese N, Pea A, Nottegar A, Smith L, Pilati C, et al. Alternative lengthening of telomeres (ALT) influences survival in soft tissue sarcomas: a systematic review with meta-analysis. BMC Cancer. 2019;19:232 This study definitively clarified the prognostic importance of ALT also in sarcomas.

12. Ferreira MSV, Sørensen MD, Pusch S, Beier D, Bouillon AS, Kristensen BW, et al. Alternative lengthening of telomeres is the major telomere maintenance mechanism in astrocytoma with isocitrate dehydrogenase 1 mutation. J Neuro-Oncol. 2020;147:114.

13. Minasi S, Baldi C, Pietsch T, Donofrio V, Pollo B, Antonelli M, et al. Telomere elongation via alternative lengthening of telomeres (ALT) and telomerase activation in primary metastatic medulloblastoma of childhood. J Neuro-Oncol. 2019;142:435-44.

14.• Marinoni I, Kurrer AS, Vassella E, Dettmer M, Rudolph T, Banz V, et al. Loss of DAXX and ATRX are associated with chromosome instability and reduced survival of patients with pancreatic neuroendocrine tumors. Gastroenterology. 2014;146:453-60.e5 First report of ALT in pancreatic neuroendocrine tumors.

15. O'Sullivan RJ, Almouzni G. Assembly of telomeric chromatin to create ALTernative endings. Trends Cell Biol. 2014;24:675-85.

16. Pea A, Yu J, Marchionni L, Noe M, Luchini C, Pulvirenti A, et al. Genetic analysis of small well-differentiated pancreatic neuroendocrine tumors identifies subgroups with differing risks of liver metastases. Ann Surg. 2020;271:566-73 Demonstration of the prognostic relevance of ALT also for small $(<3 \mathrm{~cm})$ pancreatic neuroendocrine tumors.

17. Singhi AD, Liu TC, Roncaioli JL, Cao D, Zeh HJ, Zureikat AH, et al. Alternative lengthening of telomeres and loss of DAXX/ATRX expression predicts metastatic disease and poor survival in patients with pancreatic neuroendocrine tumors. Clin Cancer Res. 2017;23:600-9.

18.• Scarpa A, Chang DK, Nones K, Corbo V, Patch AM, Bailey P, et al. Whole-genome landscape of pancreatic neuroendocrine tumours. Nature. 2017;543:65-71 Comprehensive genomic analysis of pancreatic neuroendocrine tumors.

19. Mafficini A, Scarpa A. Genetics and epigenetics of gastroenteropancreatic neuroendocrine neoplasms. Endocr Rev. 2019;40:506-36.

20. Kim HS, Lee HS, Nam KH, Choi J, Kim WH. Telomere length abnormalities and telomerase RNA component expression in gastroenteropancreatic neuroendocrine tumors. Anticancer Res. 2015;35:3501-10.

21.• Kim JY, Brosnan-Cashman JA, An S, Kim SJ, Song KB, Kim MS, et al. Alternative lengthening of telomeres in primary pancreatic neuroendocrine tumors is associated with aggressive clinical behavior and poor survival. Clin Cancer Res. 2017;23:1598-606 Important validation, using a large sample size, of ALT as a prognostic biomarker in pancreatic neuroendocrine tumors.

22. Hackeng WM, Schelhaas W, Morsink FHM, Heidsma CM, van Eeden S, Valk GD, et al. Alternative lengthening of telomeres and differential expression of endocrine transcription factors distinguish metastatic and non-metastatic insulinomas. Endocr Pathol. 2020;31:108-18 The prognostic importance of ALT has been confirmed also for pancreatic insulinomas.

23. VandenBussche CJ, Allison DB, Graham MK, Charu V, Lennon $\mathrm{AM}$, Wolfgang CL, et al. Alternative lengthening of telomeres and ATRX/DAXX loss can be reliably detected in FNAs of pancreatic neuroendocrine tumors. Cancer Cytopathol. 2017;125:544-51.

24. Dogeas E, Karagkounis G, Heaphy CM, Hirose K, Pawlik TM, Wolfgang CL, et al. Alternative lengthening of telomeres predicts site of origin in neuroendocrine tumor liver metastases. J Am Coll Surg. 2014;218:628-35.

Publisher's Note Springer Nature remains neutral with regard to jurisdictional claims in published maps and institutional affiliations. 